\title{
First Principle Study of Doping Effects (Ti, Cu, and Zn) on Electrochemical Performance of $\mathrm{Li}_{2} \mathrm{MnO}_{3}$ for Lithium-ion Batteries
}

\author{
Zahra Moradi ${ }^{1}$, Amir Heydarinasab ${ }^{1}$, and Farshid Pajoum Shariati ${ }^{1}$ \\ ${ }^{1}$ Azad University
}

July 6, 2020

\begin{abstract}
Li-rich layered Mn-based oxides (LMOs) have attracted much attention due to their potential in various applications as cathode materials with high energy density. However, these cathode materials still suffer from drawbacks such as poor rate capability and voltage decay which makes further investigation vital and rational. Herein, the doping strategy is employed to investigate the effect of $\mathrm{TM}=\mathrm{Ti}, \mathrm{Cu}$, and $\mathrm{Zn}$ on $\mathrm{Li}_{2} \mathrm{Mn}_{0.5} \mathrm{TM}_{0.5} \mathrm{O}_{3}$ for improving electrochemical performances of $\mathrm{Li}_{2} \mathrm{MnO}_{3}$. The electrochemical properties such as voltage, electrical conductivity, safety, structural stability, and kinetics and mechanism of Li-ion diffusion are evaluated and compared. All doped cathodes decrease the voltage reduction and improve the electrical conductivity coefficient in comparison with LMO. Ti dopants exhibit the potential to increase the maximum voltage of LMO and structural stability. Doping $\mathrm{Zn}$ and $\mathrm{Cu}$ elements can delay the oxygen loss which leads to a higher life cycle and safety. Also, the substitution of $\mathrm{Zn}$ dopants decreases the energy barrier against Li-ion diffusion and consequently, the lower Li-ion diffusion coefficient is expected. Using $\mathrm{Ti}, \mathrm{Cu}$, and $\mathrm{Zn}$ with $\alpha=0.5$ in $\mathrm{Li}_{2} \mathrm{Mn}_{0.5} \mathrm{TM}_{\alpha} \mathrm{O}_{3}$ may furthermore open a door for the synthesis of lithium-rich materials with enhanced performance.
\end{abstract}

\section{Hosted file}

Manuscrpit.docx available at https://authorea.com/users/340159/articles/467301-firstprinciple-study-of-doping-effects-ti-cu-and-zn-on-electrochemical-performance-ofli2mno3-for-lithium-ion-batteries 